\title{
Minimalistic Scotogenic Scalar Dark Matter
}

\author{
Valentina De Romeri \\ Institut de Física Corpuscular CSIC/Universitat de València, Parc Científic de Paterna \\ C/ Catedrático José Beltrán, 2 E-46980 Paterna (Valencia) - Spain
}

\begin{abstract}
In this talk, we reexamine the minimal Singlet + Triplet Scotogenic Model, where dark matter is the mediator of neutrino mass generation. We assume it to be a scalar WIMP, whose stability follows from the same $\mathbb{Z}_{2}$ symmetry that leads to the radiative origin of neutrino masses. We performed a full numerical analysis of the signatures expected at dark matter as well as collider experiments.
\end{abstract}

Keywords: Beyond the Standard Model, Neutrino Physics, Dark Matter, Scotogenic DOI: 10.31526/ACP.NDM-2020.18

\section{THE SINGLET + TRIPLET SCOTOGENIC MODEL}

This generalization of the scotogenic model [1] was proposed in [2] and further studied in several papers, e.g. [3, 4]. In this talk we summarise the study carried on in [5], to which we refer for more details.

In this scenario, an additional discrete $\mathbb{Z}_{2}$ symmetry has the rôle to make the lightest $\mathbb{Z}_{2}$-odd or "dark" particle stable and to ensure the radiative generation of neutrino masses. The SM particle content is extended by the inclusion of a Majorana fermion triplet $\Sigma$ and a Majorana fermion singlet $F$, both odd under the $\mathbb{Z}_{2}$ symmetry. Additionally, the model includes a new scalar doublet $\eta$ - odd under the $\mathbb{Z}_{2}$ symmetry, which does not acquire a vacuum expectation value $(\mathrm{VEV})$ - and a triplet scalar $\Omega$, which allows for the mixing of the neutral parts of the new fermions. Table 1 summarises the full particle content of the model, detailing the corresponding charge assignment under the different symmetry groups. Taking into account the new fields and symmetries of the model, the relevant terms of the La-

\begin{tabular}{|c|c|c|c|c|c|c|c|}
\hline & \multicolumn{3}{|c|}{ Standard Model } & \multicolumn{2}{c|}{ new fermions } & \multicolumn{2}{c|}{ new scalars } \\
\hline & $L$ & $e$ & $\phi$ & $\Sigma$ & $F$ & $\eta$ & $\Omega$ \\
\hline Generations & 3 & 3 & 1 & 1 & 1 & 1 & 1 \\
\hline \hline $\mathrm{SU}(3)_{\mathrm{C}}$ & 1 & 1 & 1 & 1 & 1 & 1 & 1 \\
$\mathrm{SU}(2)_{\mathrm{L}}$ & 2 & 1 & 2 & 3 & 1 & 2 & 3 \\
$\mathrm{U}(1)_{\mathrm{Y}}$ & -1 & -2 & 1 & 0 & 0 & 1 & 0 \\
$\mathbb{Z}_{2}$ & + & + & + & - & - & - & + \\
\hline $\mathrm{L}$ & 1 & 1 & 0 & 0 & 0 & -1 & 0 \\
\hline
\end{tabular}

TABLE 1: Particle content and quantum numbers of the Singlet + Triplet Scotogenic Model. The charge assignments of the fields under the global Lepton Number symmetry (L) are also shown.

grangian read

$$
\mathcal{L} \subset-Y^{\alpha \beta} L_{\alpha} e_{\beta} \phi-Y_{F}^{\alpha}\left(\bar{L}_{\alpha} \tilde{\eta}\right) F-Y_{\Sigma}^{\alpha} \bar{L}_{\alpha}^{c} \Sigma^{\dagger} \tilde{\eta}-Y_{\Omega} \operatorname{Tr}[\bar{\Sigma} \Omega] F-\frac{1}{2} M_{\Sigma} \operatorname{Tr}\left(\bar{\Sigma}^{c} \Sigma\right)-\frac{M_{F}}{2} \overline{F^{c}} F+\text { h.c. }
$$

where $\tilde{\eta}=i \sigma_{2} \eta^{*}$. The first Yukawa term $Y^{\alpha \beta}$ is the SM interaction for leptons, which we can assume to be diagonal in flavour (Greek indices stand for family indices). The scalar potential $\mathcal{V}$ invariant under the $\mathrm{SU}(2) \times \mathrm{U}(1) \times \mathbb{Z}_{2}$ symmetry is

$$
\begin{aligned}
\mathcal{V} & =-m_{\phi}^{2} \phi^{\dagger} \phi+m_{\eta}^{2} \eta^{\dagger} \eta-\frac{m_{\Omega}^{2}}{2} \operatorname{Tr}\left(\Omega^{\dagger} \Omega\right) \\
& +\frac{\lambda_{1}}{2}\left(\phi^{\dagger} \phi\right)^{2}+\frac{\lambda_{2}}{2}\left(\eta^{\dagger} \eta\right)^{2}+\frac{\lambda_{3}}{2}\left(\phi^{\dagger} \phi\right)\left(\eta^{\dagger} \eta\right)+\lambda_{4}\left(\phi^{\dagger} \eta\right)\left(\eta^{\dagger} \phi\right)+\frac{\lambda_{5}}{2}\left[\left(\phi^{\dagger} \eta\right)^{2}+\left(\eta^{\dagger} \phi\right)^{2}\right]+\mu_{1} \phi^{\dagger} \Omega \phi+\mu_{2} \eta^{\dagger} \Omega \eta \\
& +\frac{\lambda_{1}^{\Omega}}{2}\left(\phi^{\dagger} \phi\right) \operatorname{Tr}\left(\Omega^{\dagger} \Omega\right)+\frac{\lambda_{2}^{\Omega}}{4}\left[\operatorname{Tr}\left(\Omega^{\dagger} \Omega\right)\right]^{2}+\frac{\lambda_{\eta}^{\Omega}}{2}\left(\eta^{\dagger} \eta\right) \operatorname{Tr}\left(\Omega^{\dagger} \Omega\right),
\end{aligned}
$$

where we make the conservative assumption that $m_{\phi}^{2}, m_{\eta}^{2}$ and $m_{\Omega}^{2}$ are all positive, so that the spontaneous electroweak symmetry breaking will be driven by $\phi$ and (sub-dominantly) by the neutral component of $\Omega$, while $\eta$ cannot acquire a VEV. The couplings in Eq. 2 must comply with some conditions [5] in order to ensure that $\mathcal{V}$ is consistently bounded from below at the electroweak scale. However, the running of the RGEs may still lead to the breaking of the $\mathbb{Z}_{2}$ symmetry at some higher energy scale. Moreover we require that the scalar quartic couplings in Eq. 2 are $\lesssim 1$ so that the expansion of the potential $\mathcal{V}$ around its minimum is perturbatively valid. Because of the conservation of the $\mathbb{Z}_{2}$ symmetry, the $\mathbb{Z}_{2}$-odd scalar field $\eta$ does not mix with any other scalar. In terms of its CP-even and CP-odd components the $\eta$ field is: $\eta^{0}=\frac{\left(\eta_{R}+i \eta_{I}\right)}{\sqrt{2}}$. The physical masses of the neutral $\eta$ field are: $m_{\eta_{R(I)}}^{2}=m_{\eta}^{2}+\frac{1}{2}\left(\lambda_{3}+\lambda_{4}+(-) \lambda_{5}\right) v_{\phi}^{2}+\frac{1}{2} \lambda_{\eta}^{\Omega} v_{\Omega}^{2}-\frac{1}{\sqrt{2}} \mu_{2} v_{\Omega}$. The difference $m_{\eta_{R}}^{2}-m_{\eta_{I}}^{2}$ depends only on the parameter $\lambda_{5}$ which is also responsible for the smallness of neutrino masses. The lightest of the two eigenstates $\eta_{R, I}$ a viable dark matter (DM) candidate. 


\section{PHENOMENOLOGY OF SCALAR SCOTOGENIC DARK MATTER}

We assume the $\mathbb{Z}_{2}$-odd scalar $\eta_{R}$ to be the DM candidate and we investigate its phenomenology in full detail.

\subsection{Relic density}

We show in Fig. 1 the expected DM relic abundance as a function of the mass of the scalar DM candidate $\eta_{R}$. The narrow black band depicts the $3 \sigma$ range for cold DM derived by the Planck satellite data [6]. Only for solutions falling exactly in this band (cyan points) the totality of DM can be explained by $\eta_{R}$. Blue points refer to solutions where $\eta_{R}$ would be subdominant, and another DM candidate would be required. Grey points are excluded by a variety of constraints [5]. Dark grey points are solutions in conflict with the current limit on WIMP-nucleon spin-independent (SI) elastic scattering cross section set by XENON1T [7].

\subsection{Direct detection}

The tree-level SI $\eta_{R}$-nucleon interaction cross section is mediated through the Higgs and the $\mathrm{Z}$ portals. Since the $\eta$ doublet has non-zero hypercharge, the $\eta_{R}$ - nucleon interaction through the $\mathrm{Z}$ boson would in general exceed the current constraints from direct detection experiments. Nevertheless, in most solutions, $\lambda_{5}$ induces a mass splitting between the CP-odd partner $\eta_{I}$ and $\eta_{R}$ such that the interaction through the $\mathrm{Z}$ boson is kinematically forbidden, or leads to inelastic scattering. The $\eta_{R^{-}}$ nucleon interaction via the Higgs is therefore dominant in most of the parameter space. As a consequence, the coupling between $\eta_{R}$ and the Higgs boson (which depends on the sum $\lambda_{3}+\lambda_{4}+\lambda_{5}$ and on $v_{\Omega}, \mu_{2}$ and $\lambda_{\eta}^{\Omega}$ ) turns out to be the relevant quantity controlling both this cross section and the signals at LHC. We show in Fig. 2 the SI $\eta_{R}$-nucleon elastic scattering cross section weighted by $\xi=\frac{\Omega_{\eta_{R}}}{\Omega_{\text {Planck }}}$, versus the $\eta_{R}$ mass.

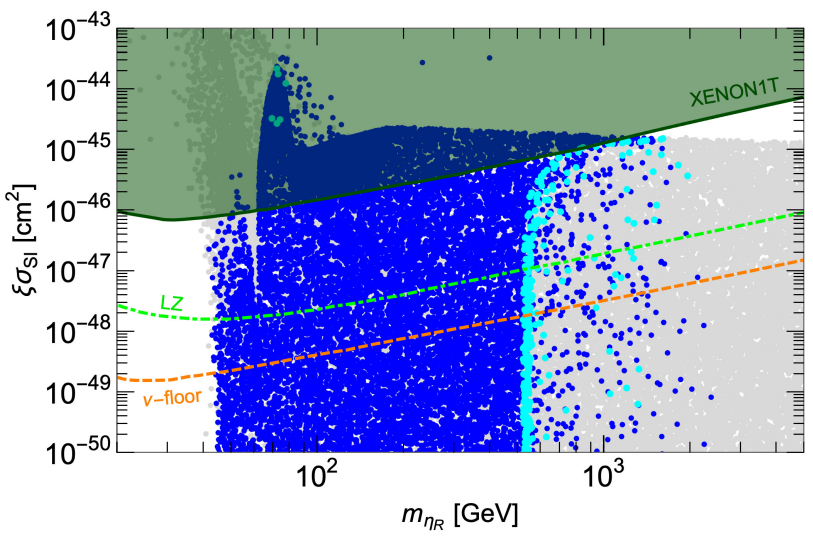

FIGURE 2: SI $\eta_{R}$-nucleon elastic scattering cross section versus the $\eta_{R}$ mass. Colour code as in Fig 1 . The dark green line denotes the most recent upper bound from XENON1T [7]. The dashed orange line corresponds to the "neutrino floor" from coherent elastic neutrino-nucleus scattering, while the green dot-dashed one stands for the projected sensitivity for LZ [8].

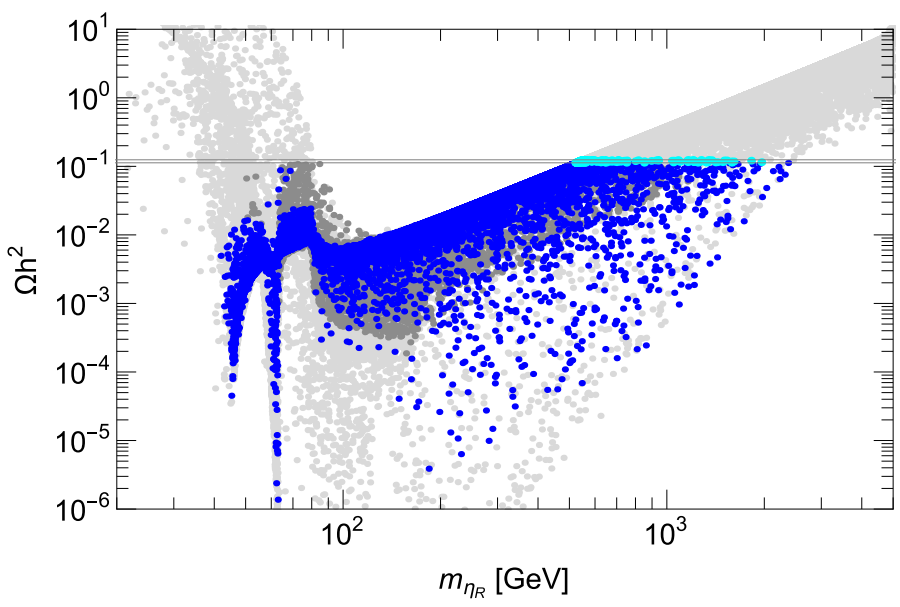

FIGURE 1: Relic abundance $\Omega_{\eta_{R}} h^{2}$ as a function of the $\eta_{R}$ mass.

\subsection{Indirect detection}

If $\eta_{R}$ annihilates into SM products with a cross section near the thermal relic benchmark value, it may be detected indirectly. Among its annihilation products, $\gamma$ rays are probably the best messengers since they proceed almost unaffected during their propagation, thus carrying both spectral and spatial information. We consider prospects of detecting $\gamma$ rays from $\eta_{R}$ annihilations by considering the continuum spectrum up to the $\eta_{R}$ mass. We assume annihilations into $b \bar{b}, \tau^{+} \tau^{-}$and $W^{+} W^{-}$to compare with current limits set by the Fermi Large Area Telescope (LAT) satellite [12] and HESS telescope [13]. Points in light red are solutions with relic abundance falling exactly within the $3 \sigma$ band measured by Planck. In the same figure we also show the 95\% C.L. upper limits currently set by the Fermi-LAT with $\gamma$-ray observations of Milky Way dwarf spheroidal satellite galaxies (dSphs) based on 6 years of data processed with the Pass 8 event-level analysis [12]. Moreover we show as a red dot-dashed curve the current upper limit 
obtained by H.E.S.S. using Galactic Center (GC) $\gamma$-ray data accumulated over 10 years [13], assuming a $W^{+} W^{-}$channel and an Einasto DM density profile. Finally, we also depict sensitivity projections for Fermi-LAT from a stacked analysis of $60 \mathrm{dSphs}$ and 15 years of data, in the $b \bar{b}$ channel [14] (dashed orange) and for CTA, for the Milky way galactic halo target, $W^{+} W^{-}$channel and an Einasto DM density profile [15]. Although current limits lie a couple of orders of magnitude above the predicted signals in this model, future data from Fermi-LAT and CTA offer promising prospects, eventually allowing one to test part of the parameter space both in the low $(\sim 70 \mathrm{GeV})$ and in the high $(\gtrsim 500 \mathrm{GeV})$ mass regions.

\section{SUMMARY AND CONCLUSIONS}

In this talk we have reexamined the generalised version of the minimal Singlet + Triplet Scotogenic Model, in which DM emerges naturally as the mediator of neutrino mass generation and its stability follows from the same $\mathbb{Z}_{2}$ symmetry also responsible for the radiative origin of neutrino masses. Notice that, while the simplest model of Ma [1] fails to be consistent over a wide range of parameters, this generalised scotogenic model is the minimal one allowing for a conserved $\mathbb{Z}_{2}$ symmetry all the way up to high mass scales [3]. We have assumed the DM to be a scalar WIMP and we have presented a detailed numerical analysis of the signatures expected at DM detectors. We have identified the regions of parameters where DM predictions are in agreement with theoretical and experimental constraints, such as those coming from neutrino oscillation data, Higgs data, DM relic abundance and direct detection searches. We have also presented expectations for near future direct and indirect detection experiments. These will further probe the parameter space of our scenario. Finally, we refer the reader to Ref. [5] for more results concerning collider signatures associated to the mono-jet channel at the LHC.

\section{ACKNOWLEDGEMENTS}

VDR acknowledges financial support by the SEJI/2018/033 grant, funded by Generalitat Valenciana and partial support by the Spanish grants: "Juan de la Cierva Incorporación" program (IJCI-2016-27736, Spanish MINECO), FPA2017-85216-P, PROMETEO/2018/165 (Generalitat Valenciana) and FPA2017-90566-REDC (Red Consolider MultiDark).

\section{References}

[1] E. Ma, Phys. Rev. D 73, 077301 (2006) doi:10.1103/PhysRevD.73.077301 [hep-ph/0601225].

[2] M. Hirsch, R. A. Lineros, S. Morisi, J. Palacio, N. Rojas and J. W. F. Valle, JHEP 1310, 149 (2013) doi:10.1007/JHEP10(2013)149 [arXiv:1307.8134 [hep-ph]].

[3] A. Merle, M. Platscher, N. Rojas, J. W. F. Valle and A. Vicente, JHEP 1607, 013 (2016) doi:10.1007/JHEP07(2016)013 [arXiv:1603.05685 [hep-ph]]

[4] M. A. Díaz, N. Rojas, S. Urrutia-Quiroga and J. W. F. Valle, JHEP 1708, 017 (2017) doi:10.1007/JHEP08(2017)017 [arXiv:1612.06569 [hep-ph]].

[5] I. M. Ávila, V. De Romeri, L. Duarte and J. W. F. Valle, arXiv:1910.08422 [hep-ph].

[6] N. Aghanim et al. [Planck Collaboration], arXiv:1807.06209 [astro-ph.CO].

[7] E. Aprile et al. [XENON Collaboration], Phys. Rev. Lett. 121, no. 11, 111302 (2018) doi:10.1103/PhysRevLett.121.111302 [arXiv:1805.12562 [astro-ph.CO]]

[8] D. S. Akerib et al. [LUX-ZEPLIN Collaboration], Phys. Rev. D 101, no. 5, 052002 (2020) doi:10.1103/PhysRevD.101.052002 [arXiv:1802.06039 [astro-ph.IM]].

[9] L. Lopez Honorez, E. Nezri, J. F. Oliver and M. H. G. Tytgat, JCAP 0702, 028 (2007) doi:10.1088/1475-7516/2007/02/028 [hep-ph/0612275].

[10] L. Lopez Honorez and C. E. Yaguna, JHEP 1009, 046 (2010) doi:10.1007/JHEP09(2010)046 [arXiv:1003.3125 [hep-ph]].

[11] M. A. Díaz, B. Koch and S. Urrutia-Quiroga, Adv. High Energy Phys. 2016, 8278375 (2016) doi:10.1155/2016/8278375 [arXiv:1511.04429 [hepph]].

[12] M. Ackermann et al. [Fermi-LAT Collaboration], Phys. Rev. Lett. 115, no. 23, 231301 (2015) doi:10.1103/PhysRevLett.115.231301 [arXiv:1503.02641 [astro-ph.HE]].

[13] H. Abdallah et al. [H.E.S.S. Collaboration], Phys. Rev. Lett. 117, no. 11, 111301 (2016) doi:10.1103/PhysRevLett.117.111301 [arXiv:1607.08142 [astro-ph.HE]].

[14] E. Charles et al. [Fermi-LAT Collaboration], Phys. Rept. 636, 1 (2016) doi:10.1016/j.physrep.2016.05.001 [arXiv:1605.02016 [astro-ph.HE]].

[15] B. S. Acharya et al. [CTA Consortium], doi:10.1142/10986, 10.3204/PUBDB-2020-00341 arXiv:1709.07997 [astro-ph.IM]. 